\title{
Article \\ Mechanism Analysis of the Influence of Seat Attributes on the Seat Dip Effect in Music Halls
}

\author{
Hequn Min *(D) and Yitian Liao \\ Key Laboratory of Urban and Architectural Heritage Conservation, Ministry of Education, School of Architecture, \\ Southeast University, 2 Sipailou, Nanjing 210096, China; 220180134@seu.edu.cn \\ * Correspondence: hqmin@seu.edu.cn
}

\begin{abstract}
The seat dip effect (SDE) is an acoustic phenomenon of low-frequency band attenuation that occurs in the music halls when the sound of the music passes at a near grazing incidence over the seats. In this paper, the numerical simulations on the basis of the finite element method are conducted to study the influence of seat attributes (seat height, seat spacing and seat absorption) on the SDE and the corresponding mechanism. The mapping of sound spatial distribution related to the SDE is employed to observe the behavior of sound between the seats. The results show that the dip frequency of the SDE can be shifted to frequencies lower than theoretical values when the seat height is smaller than the seat spacing. Additionally, the SDE attenuation can be distinctly suppressed in a sequence from the front seats to the rear seats with an absorption improvement to the seat back or cushion, and the seat back absorption is more effective than the cushion absorption. A mechanism analysis reveals that the SDE is highly associated with standing waves inside the seat gaps and with the "diffusion" effect on the grazing incident waves by energy flow vortexes around the top surfaces of the seats.
\end{abstract}

Keywords: seat dip effect; seat height; seat spacing; sound absorption; mechanism

Citation: Min, H.; Liao, Y. Mechanism Analysis of the Influence of Seat Attributes on the Seat Dip Effect in Music Halls. Appl. Sci. 2021, 11,9768. https://doi.org/10.3390/ app11209768

Received: 30 June 2021

Accepted: 18 October 2021

Published: 19 October 202

Publisher's Note: MDPI stays neutral with regard to jurisdictional claims in published maps and institutional affiliations.

Copyright: (c) 2021 by the authors. Licensee MDPI, Basel, Switzerland. This article is an open access article distributed under the terms and conditions of the Creative Commons Attribution (CC BY) license (https:// creativecommons.org/licenses/by/ $4.0 /)$.

\section{Introduction}

In music halls, when sound passes at a near grazing incidence over the seats, there is a phenomenon of excessive attenuation in the low-frequency band. Different from the sound absorption attenuation that usually occurs at high frequencies caused by sound being absorbed by the audience and the surfaces of the seats, this attenuation phenomenon, known as the seat dip effect (SDE), occurs at about $100 \mathrm{~Hz}$ and seems typical in music halls despite the difference made to the sound by the audience.

The effect was first discovered by two studies through investigations in concert halls and scale models [1,2], early in 1964. Subsequently, in situ or scale-model measurements [1-7], and theoretical calculations [8] were extensively conducted to analyze the SDE. It was shown that the SDE caused the excessive attenuation of sound at low frequencies within the hall, which significantly affected spatial impression [9], clarity [3], and timbre $[4,10]$ by affecting the early reflection of the sound, and people's perception of bass frequencies was significantly stronger in concert halls with higher SDE attenuation frequencies [11]. Recently, computer simulations were applied to study the SDE with the wave-based geometrical acoustics method, the finite element method (FEM), the boundary element method (BEM) [12] and the finite-difference time-domain method [13]. The parameters with a possible influence on the SDE were investigated, such as the floor inclination [5], sound incident angle [4,14], and ceiling height [4,7]. Based on these investigations, some guidelines were proposed to reduce the SDE, such as: (1) increasing the ceiling reflection or the side reflections [15,16]; (2) installing ground sound absorbers between the seat rows [3,8]; and (3) sloping the floor or the stage [5,14], etc.

However, for the SDE suppression, choosing the appropriate seat attributes of height, spacing and absorption is a more implementable way than raising the floor slope or 
adding reflectors, and seat attribute adjustment can be implemented easily in renovations which affect the hall construction. Regarding the influence of seat attributes on the SDE and possible mechanism, early studies [1,2] showed that the frequency of the maximum attenuation mainly depended on the height of the seat. Later, Ishida's measurements [17] investigated the influence on the SDE from the seat underpass and observed that the dip frequency increased for higher source positions when the height of the seat underpass decreased. Measurements and practical calculations suggested that the SDE was affected by the diffraction on the seat top [3] and by the reflected sound on the auditorium seats [14]. Davies and Cox [12] compared the SDE attenuation with different seat absorptions and seat underpass shapes using the BEM simulation and a scale model and reported that changing the seat absorption had an audible influence on reducing the SDE in concert halls. Tahvanainen et al. [18] conducted scale-model experiments to study the effect of the seat underpass and floor inclination on the SDE, and found that the dip frequency depended on the seat back height and on the obstruction degree of the seat underpass. However, little investigation and discussion on the possible mechanism was conducted in [12,18]. Takahashi [7] investigated the SDE phenomena via scale model measurements and derived a simple analytical model for attempting to explain the SDE mechanism. In his study, the SDE attenuations with different seat back absorptions were investigated and it was found that the seat backs affected the SDE attenuation and dip frequency shift. However, it was difficult for Takahashi to further explore the mechanism due to the limited simulation techniques of that time and only assumption was made that the SDE was highly related to the interference between a direct wave and reflected waves from the seats. At present, sound wave behaviors around the seats regarding the mechanisms of influence from the seat attributes of height, spacing, and absorption on the SDE are not yet clear in the literature; however, this influence is important for the reduction in the SDE by choosing appropriate seat attributes.

Given the literature review above, there is a distinct lack of detailed investigation and mechanism discussion focused on the influence of seat attributes on the SDE, especially from the analysis of the corresponding sound wave behavior around the seats. In this paper, the quantitative attenuation and mapping of sound spatial distribution related to the SDE is evaluated in simulations with the FEM to study the influence of seat height, seat spacing and seat absorption on the SDE, as well as the corresponding mechanism.

\section{Simulation}

From previous studies $[7,15]$, the reflections from walls and ceilings in a room can mask the SDE phenomenon, and the SDE is substantially related to the sound reflections from the seats and the floor. To simplify the problem and highlight the SDE phenomenon, a twodimensional geometry with simplified acoustic boundary conditions, shown in Figure 1, was established to represent the section plane of classical music halls in the present study. The simulations were carried out with the acoustic FEM in the frequency domain and were implemented with the commercial software, COMSOL Multiphysics ${ }^{\circledR}$ [19].

In the numerical model geometry in Figure 1a, the room is defined as $24.5 \mathrm{~m}$ long and $4.5 \mathrm{~m}$ high with four boundaries (ground, ceiling, front wall, and back wall), containing 16 rows of simplified seats with the height $h$ and the spacing $w$ (representing the seat pitch in actual halls). A point source $S$ is $4.5 \mathrm{~m}$ away from the front wall, at a height of $2.5 \mathrm{~m}$ above the ground, and radiates with a strength of $1 \mathrm{~W} / \mathrm{m}^{2}$. The receiving points R1-R6 are in the middle of the seat gap, at a height of $1.1 \mathrm{~m}$ above the ground. To simplify the problem, we assumed the ground to be perfectly reflecting with infinite impedance, and assumed the front wall, back wall and ceiling to be perfectly absorbing without sound reflections from these boundaries (which were implemented as Perfectly Matched Layers (PML) in numerical models) ensuring that the reflections were only from the ground and the seats to emphasize the SDE. The seats were simplified to vertical slats in the model. This configuration was used in previous scale models [2,4] and calculation models [7], while the seat sound absorption could not be considered in the former plywood models 
and the sound field distribution and acoustic behavior between the seat gaps was not observed in the latter calculation model. In the numerical models in this paper, the seat back or the floor can be defined as rigid or with a different acoustic impedance. For the investigations of the seat height and seat spacing, the seats were set as rigid boundaries to separate the influence from the seat sound absorption. For investigations on the seat-back sound absorption, the seats were set with different acoustic impedances. It should be noted that the influence from the seat cushion sound absorption on the SDE was investigated by applying different impedances on the ground between the seats in the simplified model. In the present study, the surface sound absorption was illustrated by the normal incidence sound absorption coefficient $\alpha$ (seat back absorption by $\alpha_{\mathrm{b}}$ and the seat cushion absorption by $\left.\alpha_{c}\right)$.

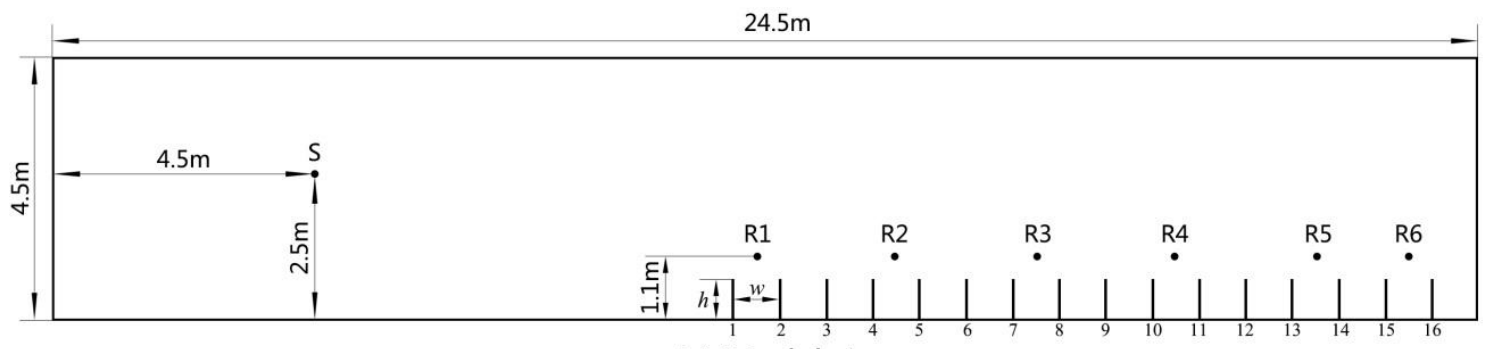

(a) Model size

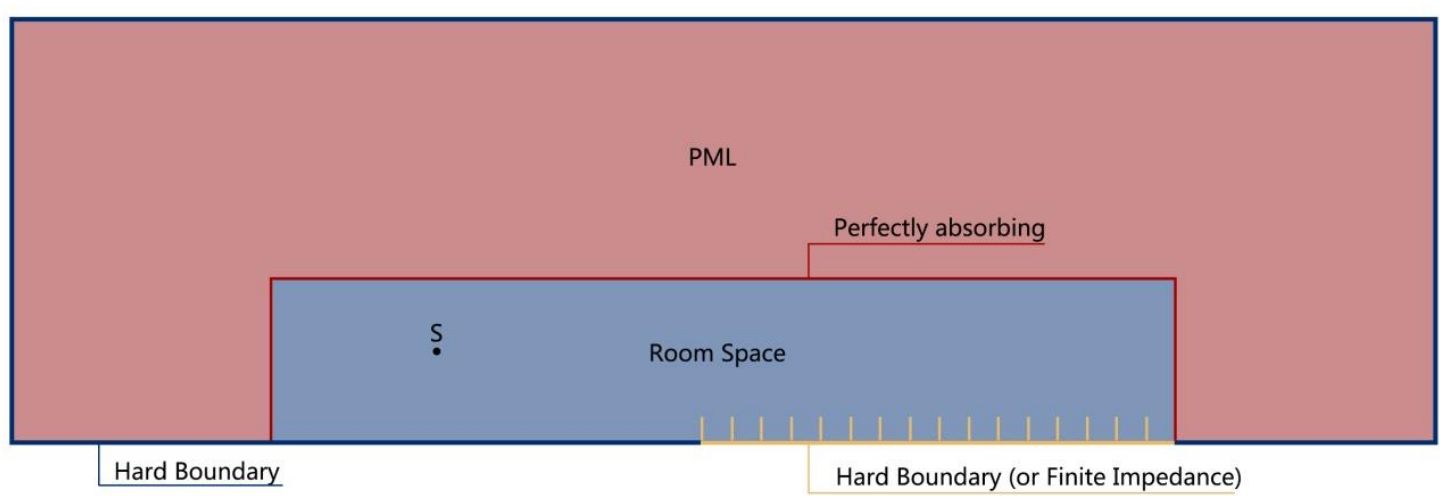

(b) Acoustic boundary conditions

Figure 1. Setup of the simulation model: (a) geometry and (b) acoustic boundary conditions.

A quantity of Relative Level $(R L)$ was applied for the investigations on the characteristics of the SDE, which are defined as:

$$
R L=S P L_{2}-S P L_{1}
$$

where $S P L_{2}$ and $S P L_{1}$ are the Sound Pressure Levels (SPLs) at each receiver when there are seats and no seats, respectively.

At first, the numerical model was compared with the measurements of a $1 / 10$ scalemodel from Sessler and West [2] for validation. In the validation case, the geometry dimensions in the simulation were set to equal values of those in the scale-model (as shown in Figure 9 in Ref. [2]) and the sound absorption arrangement of the seats and floor in the simulation was set according to the scale-model conditions $[2,8]$, where the stage and the ground were set as rigid surfaces, and the seats were set as absorptive surfaces with a specific acoustic admittance of 0.01 . The $R L$ results of the simulation and measurements at a receiver of $1.6 \mathrm{~m}$ from the sound source in this validation case were compared in Figure 2 . A good agreement can be observed between the simulation and measurements, except that in the measurements the $R L$ dip was not identified as clearly as it was in the simulation due to the limited measurement data. This shows the validity of numerical models for the further SDE investigations in this paper. 


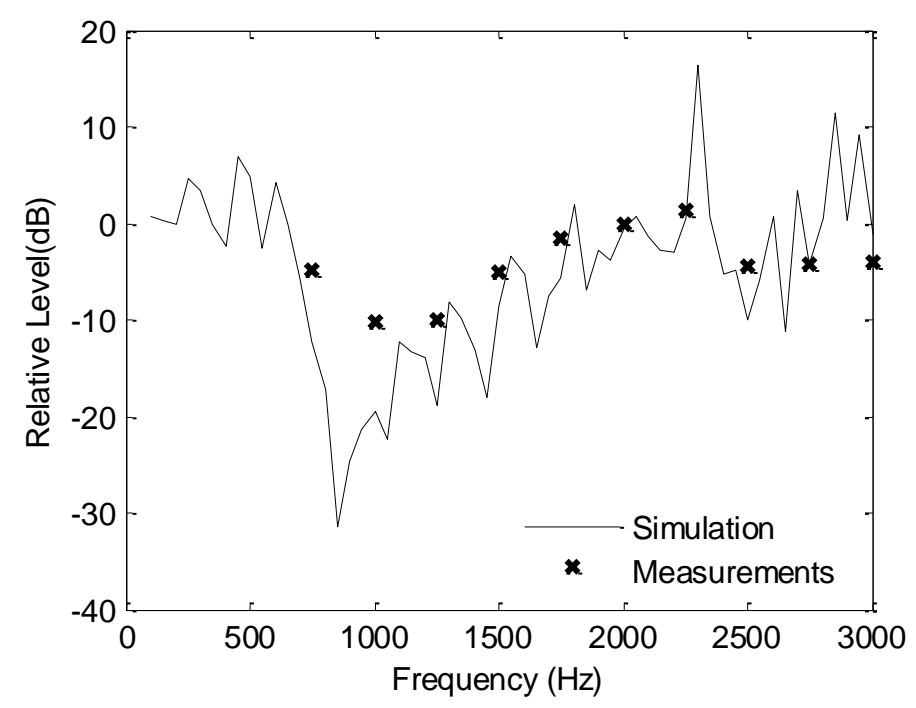

Figure 2. Comparison between the $R L$ spectra of simulation and measurements in the validation case. X-marker: measurements in the 1/10 scale model (after Sessler and West [2]). Solid line: simulation with the geometry and boundary conditions in the scale model.

Figure $3 \mathrm{a}, \mathrm{b}$ show the initial results of the $R L$ spectra and SPL spatial distribution associated with the SDE when initially $h=0.7 \mathrm{~m}, w=0.8 \mathrm{~m}$, and the seats and ground are set as rigid boundaries in simulations. In Figure $3 a$, the dip minimum of the $R L$ is close to $-30 \mathrm{~dB}$ at the dip frequency of $105 \mathrm{~Hz}$, indicating an SDE phenomenon which is the same as the previous observations [1,2]. In Figure $3 b$, the color map of SPL spatial distribution at the dip frequency of $105 \mathrm{~Hz}$ shows that the SDE phenomenon appears around the top opening of seat gaps (emphasized in dotted boxes) with distinctly low SPLs, which should be the focus area on the SDE in acoustic design. In the previous studies mentioned above, the SDE was usually analyzed only through $R L$ spectra similar to those shown in Figure 3a. In this study, the color maps similar to Figure $3 b$ at interesting frequencies were applied to observe the two-dimensional SPL attenuation associated with the SDE for further mechanism analysis. In the following, the relationships between three factors (seat height $h$, seat spacing $w$, and the seat sound absorption coefficient $\alpha$ ) and the SDE are investigated through the dip frequency $f_{0}$, the dip minimum $R L_{0}$ and the SPL spatial distribution for a further detailed analysis of the SDE mechanism, and for the possible elimination methods for the SDE in the acoustic design stage. 


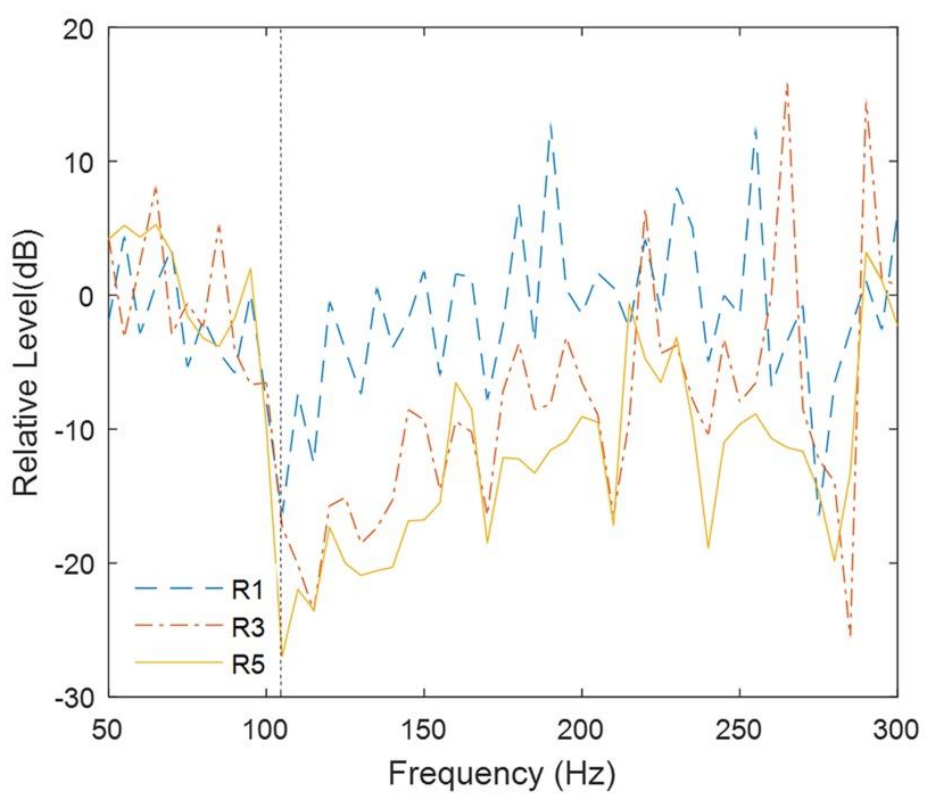

(a) SDE curves

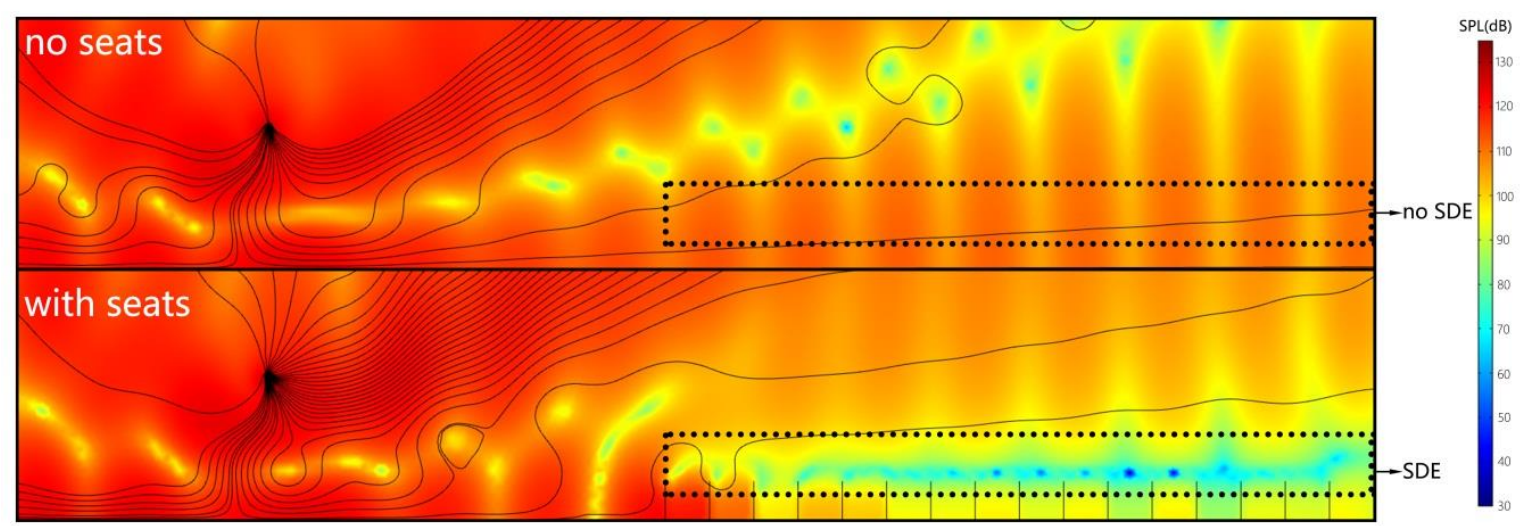

(b) SDE spatial distributions

Figure 3. Illustration of the SDE: (a) relative level spectra; (b) color maps of the SPL spatial distributions at a dip frequency $f_{0}$ of $105 \mathrm{~Hz}$. Seats and floor are rigid.

\section{Results and Discussion}

\subsection{Seat Height and Seat Spacing}

In previous classical studies [1,2], when $h \approx \lambda / 4$, where $\lambda$ represents the sound wavelength at a specific frequency, the SPLs at the receivers well above the surface of the seat top were measured to be lowest and the corresponding specific frequency was just the dip frequency $f_{0}$. This was caused by the classical interference between the grazing direct sound and the vertically reflected sound waves inside the seat gaps near the surface of the seat top. In the first numerical case, investigations were carried out with different seat heights with a fixed seat spacing of $0.8 \mathrm{~m}$ to check the SDE phenomenon. Figure 4a presents the corresponding results of the average $R L$ at receivers $R 4-R 6$, where the SDE phenomenon was more distinct. When $h$ is $1.1 \mathrm{~m}, 0.9 \mathrm{~m}, 0.7 \mathrm{~m}$ and $0.5 \mathrm{~m}$, the theoretical $f_{0}$ should be $80 \mathrm{~Hz}, 95 \mathrm{~Hz}, 120 \mathrm{~Hz}$ and $170 \mathrm{~Hz}$, respectively, according to the quarter wavelength theory above. For comparisons, as shown in Figure $4 \mathrm{a}$, the corresponding $f_{0}$ in the simulations was about $80 \mathrm{~Hz}, 90 \mathrm{~Hz}, 110 \mathrm{~Hz}$ and $130 \mathrm{~Hz}$, respectively, and was close to the theoretical values but had a noticeable shift to lower frequencies when the seat height $h$ became smaller than the seat spacing $w$. 

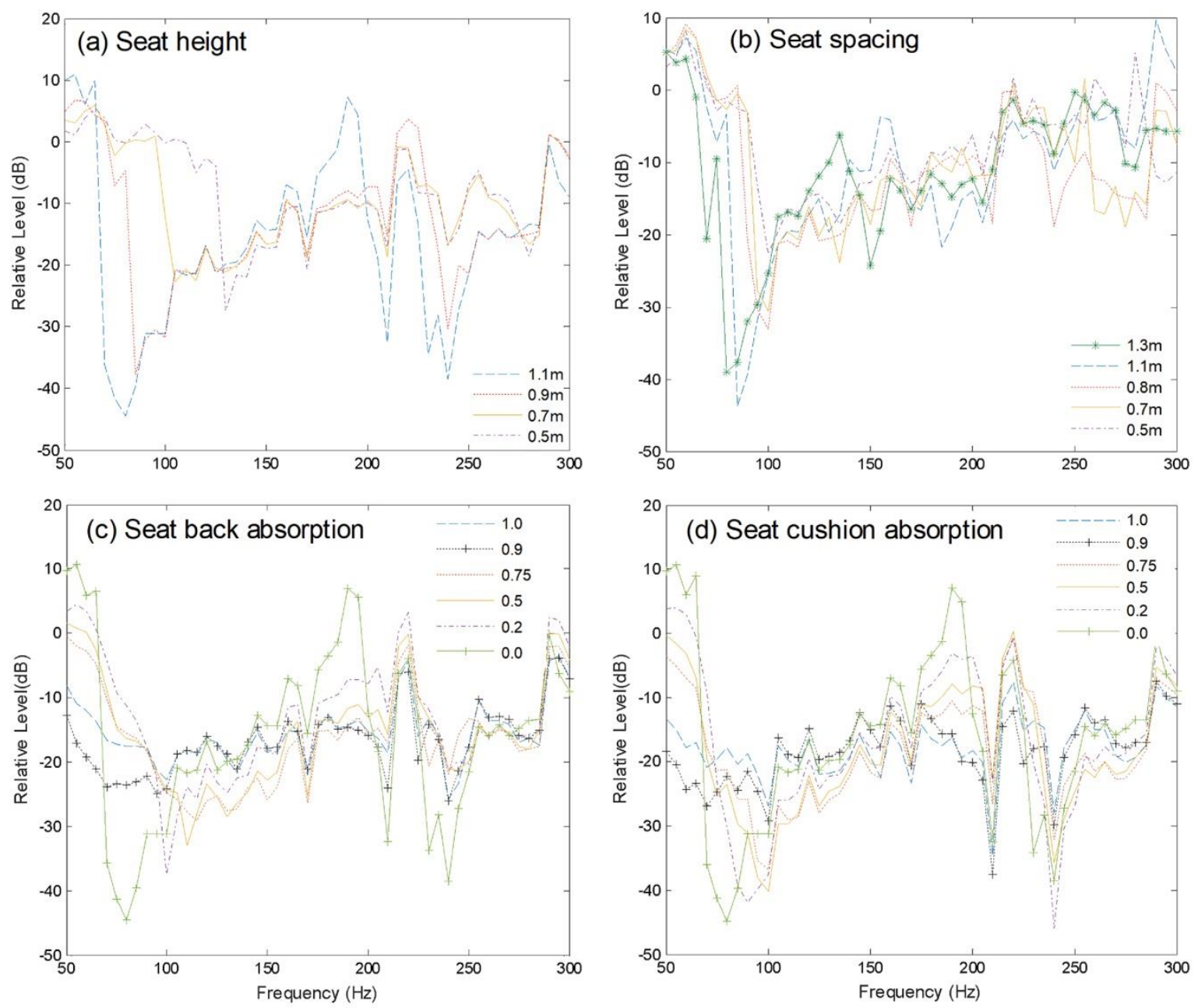

Figure 4. Spectra of average $R L$ at receivers R4-R6 for investigation of influence on the SDE from (a) seat heights $(w=0.8 \mathrm{~m}$, seats and floor are rigid); (b) seat spacing $(h=0.8 \mathrm{~m}$, seats and floor are rigid); (c) seat back absorption $(h=1.1 \mathrm{~m}$ and $w=0.8 \mathrm{~m})$ and $(\mathbf{d})$ seat cushion absorption $(h=1.1 \mathrm{~m}$ and $w=0.8 \mathrm{~m})$.

Another numerical case was conducted on the SDE phenomenon at a different seat spacing $w$ with a fixed seat height of $0.8 \mathrm{~m}$, results of which are presented in Figure $4 \mathrm{~b}$. It was shown that, when $w$ was $0.5 \mathrm{~m}, 0.7 \mathrm{~m}$ and $0.8 \mathrm{~m}$, the simulated $f_{0}$ was almost the same with a theoretical value of $105 \mathrm{~Hz}$ for the seat height $h$ of $0.8 \mathrm{~m}$, but when $w$ was $1.1 \mathrm{~m}$ or $1.3 \mathrm{~m}$, and thus larger than the seat height, a noticeable shift to a lower frequency of $85 \mathrm{~Hz}$ or $80 \mathrm{~Hz}$ was observed, respectively, as well as those in the first numerical case above. This phenomenon indicates that, when the seat height $h$ becomes smaller than the seat spacing $w$, the dip frequency $f_{0}$ may be shifted to lower frequencies compared to values from the quarter wavelength theory $[1,2]$. This may be caused by the interference between the horizontal reflections of the oblique direct sound on the seat backs. As the seat height becomes smaller or the seat spacing larger, the oblique direct sound from the source that can travel into the seat gaps becomes stronger, and leads to stronger horizontal reflections on the seat backs, and thus a stronger interference between those reflections, whose interference frequency is controlled by the seat spacing $w$. When $h$ is smaller than $w$, the interference between the horizontal reflections becomes stronger than the interference between the grazing direct sound and vertical reflections, where the frequency of the former interference is clearly lower than that of the latter interference. This leads to the observed lower frequency shift of dip frequency $f_{0}$ in this situation, and can also explain the $R L_{0}$ decreasing with $w$ increasing, as shown in Figure $4 \mathrm{~b}$. 


\subsection{Seat Absorption}

In the numerical cases above, the SDE phenomenon is investigated with rigid seats and a rigid floor. It is interesting to investigate what occurs when the seats become sound absorbing. Davies and Cox [12] conducted a rough observation on the SDE with several seat absorption coefficients but did not analyze in detail the corresponding strength and characteristic changes of the SDE. In this section, numerical cases are carried out for the SDE with a different seat back absorption coefficient $\alpha_{\mathrm{b}}$ and seat cushion absorption coefficient $\alpha_{c}$ where $h=1.1 \mathrm{~m}$ and $w=0.8 \mathrm{~m}$. The corresponding $R L$ spectrum results are presented in Figure $4 c, d$, respectively. It can be observed that, through increasing the seat back or cushion absorption coefficient from 0.0 to 1.0 , the dip minimum $R L_{0}$ gradually changes from $-46 \mathrm{~dB}$ to around $-23 \mathrm{~dB}$, demonstrating that the SDE attenuation is remarkably weakened by the seat absorption. It is also shown that, the dip frequency $f_{0}$ may increase to higher frequencies with a higher seat absorption, especially at the seat back. A similar phenomenon was observed by Takahashi [7], whose complete mechanism is unclear yet and can be related to sound distribution around the vertical slats that are assumed as simplified geometry of seat backs [7].

For a direct visual inspection on the influence of seat absorption, the color maps of the SPL spatial distribution at the corresponding dip frequencies of the SDE with different $\alpha_{b}$ or $\alpha_{c}$, as shown in Figure 4c,d, are presented in Figure 5. Figure 5a,b show the situations of seat back absorption and seat cushion absorption, respectively. In the color maps, the color represents the SPL, and the streamline is derived from the gradient of the calculated sound intensity. As shown in Figure 5, there is no SDE when no seat is in the room. When the rigid seats are set, there is a distinct area of the SDE concentrated at almost the entire surface of the seat tops, with a continuous attenuation of sound energy, and the SDE area becomes larger at seat locations farther away from the source. In Figure $5 \mathrm{a}$, as $\alpha_{\mathrm{b}}$ increases, the concentration strength of the sound energy attenuation is gradually weakened and the area of the sound energy attenuation gradually shifts backwards from the front seats. This shows that the diminishing tendency of the SDE by seat absorption is from the front seats to the rear seats. Therefore, the SDE attenuation investigated at the R4-R6 receiving points (whose positions are marked by black stars in each color map) decreases as $\alpha_{b}$ increases from 0.0 to 0.9 , as shown in Figure 4c. Meanwhile, the streamlines in Figure 5a also gradually extend into the seat gaps, showing that more sound energy flows into the seating area with a higher seat absorption. When $\alpha_{b}=1.0$, the SDE attenuation around the surface of the seat tops becomes much weaker in comparison to the situation with no seats.

Figure $5 \mathrm{~b}$ presents a similar diminishing tendency of the SDE by seat cushion absorption, but the corresponding SDE reduction is observed to not be as distinct as the reduction by the seat back absorption shown in Figure 5a. Through a cross comparison between the results in Figure 5a,b, it is shown that the appropriate sound absorption treatment of the seat back or cushion can play a certain role in suppressing the SDE in music halls, and the seat back absorption is more effective than the seat cushion absorption. From other extensive simulation cases, when $h$ is not larger than $w$, similar results (not presented here for conciseness) also show a greater beneficial effect on the SDE from the seat back absorption rather than from the seat cushion absorption, and this observation is consistent with discussion presented in Ref. [12]. The above results also suggest that common sound absorption does not have a distinct SDE reduction effect in rear seat areas, but the high sound absorption treatment does. For real applications, it is thought that a high sound absorption treatment at low frequencies on seats could be achieved by filling the appropriate metamaterial absorbers $[20,21]$ into the seat back or cushion. Additionally, in real music halls with audience, it is also suggested that the occupied seats with equivalent absorption should have suppression effects on the SDE. However, for the influence of seat occupancy on the SDE, further study is needed for a confirmation with human models on the seats and will be reported in the future. 


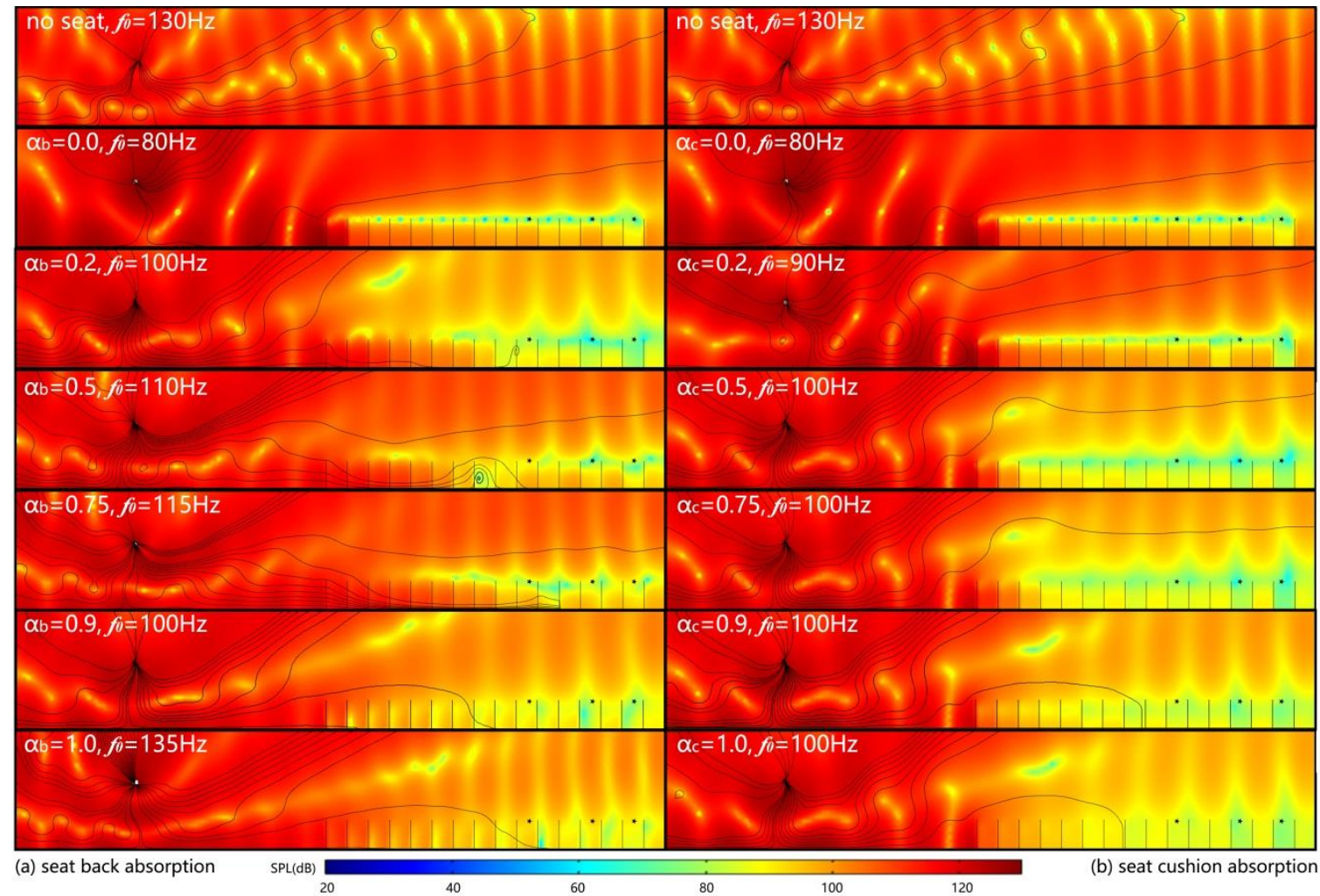

Figure 5. Comparison of SPL distributions at corresponding different dip frequencies with different sound absorption coefficients of seat backs (a): no seat; $\alpha_{b}=0.0 ; \alpha_{b}=0.2 ; \alpha_{b}=0.5 ; \alpha_{b}=0.75 ; \alpha_{b}=0.9 ; \alpha_{b}=1.0$; and of seat cushions (b): no seat; $\alpha_{c}=0.0 ; \alpha_{c}=0.2 ; \alpha_{c}=0.5 ; \alpha_{c}=0.75 ; \alpha_{b}=0.9 ; \alpha_{c}=1.0$.

\subsection{Possible Mechanism}

To clarify the influence mechanism of the seat attributes on the SDE mentioned above, two sets of detailed investigations on sound wave behavior between the seats were conducted in simulations on the possible mechanism related to the SDE. For the first investigation set, the color map results of the SPL and sound intensity (SI) distribution at different frequencies inside the rigid seat gaps are presented in Figure 6a-c where $h=0.8 \mathrm{~m}$, $w=1.1 \mathrm{~m}$ and in Figure $6 \mathrm{~d}-\mathrm{f}$ where $h=1.1 \mathrm{~m}, w=0.8 \mathrm{~m}$. The color represents the SPL, and the arrows represent the SI magnitude and direction. In Figure $6 \mathrm{a}-\mathrm{f}$, the first phenomenon is that the SI magnitudes gradually decrease from the outside to the inside of the seat gaps, and the SI directions rotate clockwise between the seats and create energy flow vortexes showing the presence of standing waves, as reported by Schultz [1]. The lowest SPL position always appears at the gap center with the height of $\lambda / 4$, which is the center point of the energy flow vortex shown in Figure 6a-e with heights of $0.81 \mathrm{~m}, 0.65 \mathrm{~m}$, and $0.57 \mathrm{~m}$ at the frequencies of $105 \mathrm{~Hz}, 130 \mathrm{~Hz}, 150 \mathrm{~Hz}$, respectively. It is worth noting that Figure $6 \mathrm{f}$ features the two lowest SPL positions at the gap center with heights of $0.35 \mathrm{~m}$ and $1.06 \mathrm{~m}$, which are equal to the $\lambda / 4$ and $3 \lambda / 4$ of the investigated frequency, $240 \mathrm{~Hz}$, respectively. This phenomenon of the lowest SPL positions inside the seat gaps located at the gap center, with the height $((2 \mathrm{k}+1) \lambda) / 4(\mathrm{k}=0,1,2 \ldots)$, shows the first influence mechanism on the SDE from seat height and spacing. The second phenomenon is that, when the energy flow vortexes appear near the top surface of the seats, for example the vortexes near the slats' upper ends, as shown in Figure $6 \mathrm{a}, \mathrm{b}, \mathrm{f}$, the grazing incident waves are observed to be "diffused" by the energy flow vortexes and a continuous attenuation can be created around the top surface of the seats due to this complex interference. This can be another influence mechanism on the SDE from seat height and spacing. These phenomena come not only from the interference between the grazing direct sound and the vertically 
reflected sound waves inside the seat gaps, but also from those between the oblique sound incidence and horizontal sound reflections inside the seat gaps.
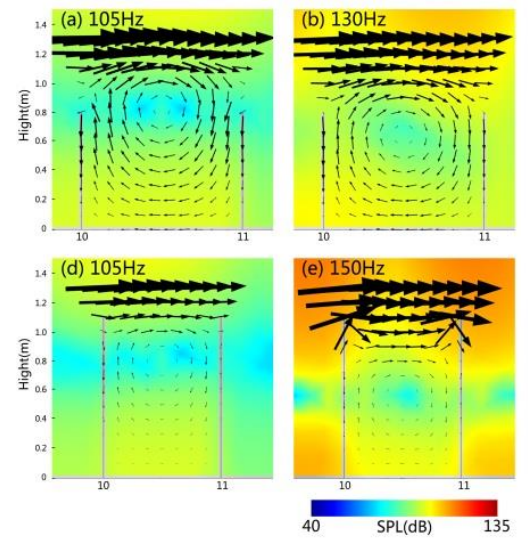
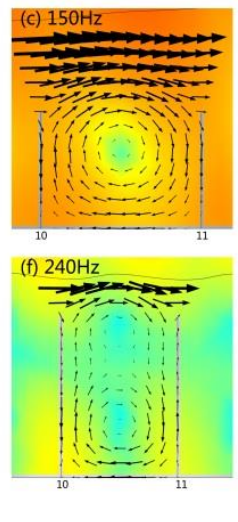
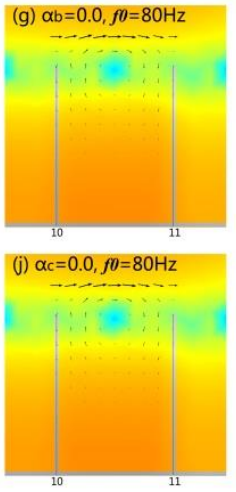

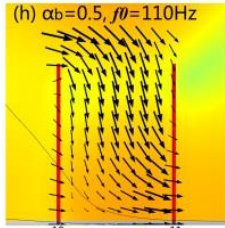

(k) $\alpha_{\mathrm{c}}=0.5, f 0=100 \mathrm{~Hz}$

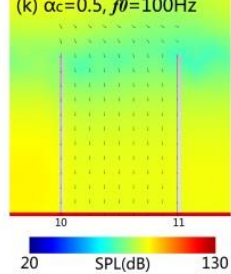

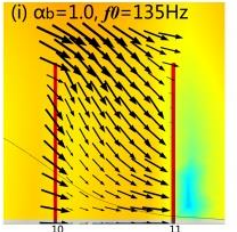

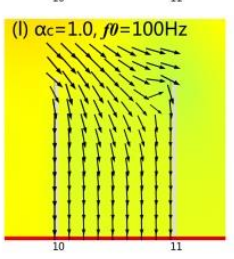

Figure 6. The SPL and SI distribution inside seat gaps: (a-c) seats are rigid, $h=0.8 \mathrm{~m}$, and $w=1.1 \mathrm{~m}$; (d-f) seats are rigid, $h=1.1 \mathrm{~m}$, and $w=0.8 \mathrm{~m} ;(\mathbf{g}-\mathbf{i}) \alpha_{\mathrm{c}}=0.0, h=1.1 \mathrm{~m}$, and $\left.w=0.8 \mathrm{~m} ; \mathbf{j}-\mathbf{l}\right) \alpha_{\mathrm{b}}=0.0, h=1.1 \mathrm{~m}$, and $w=0.8 \mathrm{~m}$.

For the second investigation set, the color map results of the SPL and SI distribution at the corresponding dip frequencies are presented in Figure 6g-I, where $\alpha_{c}=0.0, h=1.1 \mathrm{~m}$ and $w=0.8 \mathrm{~m}$ for the influence of the seat back absorption and Figure $6 \mathrm{j}-1$, where $\alpha_{\mathrm{b}}=0.0$, $h=1.1 \mathrm{~m}$, and $w=0.8 \mathrm{~m}$ for the influence of the seat cushion absorption. The absorptive seat boundaries (backs or cushions) are marked with thick red lines, while the rigid ones are marked with thick grey lines. It is shown that seat absorption has a clear guiding effect on the SI directions, where sound energy flows are observed to be guided by the absorption to become horizontal into seat backs, in Figure $6 \mathrm{~g}-\mathrm{I}$, or to become vertical into seat cushions, in Figure 6j-1. Additionally, inside the seat gaps, the horizontal reflections are distinctly reduced by the seat back absorption, as shown in Figure $6 \mathrm{~h}-\mathrm{i}$, and vertical reflections are distinctly reduced by the seat cushion absorption, as shown in Figure 6k-l. The wave guiding effect and standing wave suppression from the seat absorption remarkably level out the energy distribution and weaken the energy flow vortexes due to the complex interference inside the aforementioned seat gaps, and then reasonably suppress the SDE. This could be the influence mechanism on the SDE from the seat back or cushion absorption. Moreover, since the SDE is governed by the interference among the grazing direct sound and vertical reflection when $h$ is larger than $w$, as discussed in Section 3.1, from this mechanism it is explicable that the seat back absorption is more effective than the cushion absorption on the SDE reduction in this situation, as shown in Figure 5. The reason for this could be that the seat back absorption guides many vertical energy flows to become horizontal and then effectively weakens the interference governing the SDE in this situation.

\section{Conclusions}

In this paper, the influence of seat attributes (seat height, spacing, and seat absorption) on the SDE in music halls, as well as its possible mechanism, are studied in FEM simulations with a simplified geometry. The mapping of the SPL and SI distribution is employed to observe the spatial characteristics of the SDE for analysis. The results show that, when the seat height is smaller than the seat spacing, there is a noticeable shift of the dip frequency from the theoretical values to the lower ones. It is also shown that, with the absorption improvement of the seat back or cushion, the SDE attenuation can be suppressed in sequence from the front seats to the rear seats, and the seat back absorption is more effective than the cushion absorption. The mechanism analysis, by observing in detail the wave behavior between the seats, reveals that the standing waves inside the seat gaps act as the main cause of the SDE and the "diffusion" effect on the grazing incident waves by the energy flow vortexes around the top surface of the seats may act as another cause. The high sound absorption treatment of the seats can distinctly weaken the SDE phenomenon for 
their effective use of reducing the standing waves. In the future work, human models will be added to further study the influence of seat occupancy on the SDE. The work in this paper can provide useful insights and suggestions for the better understanding and suppression of the SDE in actual music halls.

Author Contributions: Conceptualization, H.M.; methodology, H.M. and Y.L.; software, H.M. and Y.L.; validation, H.M. and Y.L.; formal analysis, H.M.; investigation, H.M. and Y.L.; resources, H.M.; data curation, H.M.; writing-original draft preparation, H.M. and Y.L.; writing-review and editing, H.M.; visualization, Y.L.; supervision, H.M.; project administration, H.M.; funding acquisition, H.M. All authors have read and agreed to the published version of the manuscript.

Funding: This research was funded by the Natural Science Foundation of China, grant number 51408113 and the Natural Science Foundation of Jiangsu Province, China, grant number BK20140632.

Institutional Review Board Statement: Not applicable.

Informed Consent Statement: Not applicable.

Data Availability Statement: Not applicable.

Conflicts of Interest: The authors declare no conflict of interest. The funders had no role in the design of the study; in the collection, analyses, or interpretation of data; in the writing of the manuscript, or in the decision to publish the results.

\section{References}

1. Schultz, T.J.; Watters, B.G. Propagation of Sound across Audience Seating. J. Acoust. Soc. Am. 1964, 36, 885-896. [CrossRef]

2. Sessler, G.M.; West, J.E. Sound transmission over theatre seats. J. Acoust. Soc. Am. 1964, 36, 1725-1732. [CrossRef]

3. Davies, W.; Lam, Y. New attributes of seat dip attenuation. Appl. Acoust. 1994, 41, 1-23. [CrossRef]

4. Bradley, J.S. Some further investigations of the seat dip effect. J. Acoust. Soc. Am. 1991, 90, 324. [CrossRef]

5. West, J.E.; Sessler, G.M. Model Study of the Sound Transmission over Raked Theatre Seats. J. Acoust. Soc. Am. 1966, $40,1246$. [CrossRef]

6. Greenberg, D. Seat-dip phenomenon. J. Acoust. Soc. Am. 1994, 96, 3267. [CrossRef]

7. Takahashi, D. Seat dip effect: The phenomena and the mechanism. J. Acoust. Soc. Am. 1997, 102, 1326-1334. [CrossRef]

8. Ando, Y.; Takaishi, M.; Tada, K. Calculation of the sound transmission over theater seats and methods for its improvement in the low-frequency range. J. Acoust. Soc. Am. 1982, 72, 443-448. [CrossRef]

9. Barron, M.; Marshall, A. Spatial impression due to early lateral reflections in concert halls: The derivation of a physical measure. J. Sound Vib. 1981, 77, 211-232. [CrossRef]

10. Davies, W.J.; Cox, T.J.; Lam, Y.W. Subjective Perception of Seat-dip Attenuation. Acta Acust. United Acust. 1996, 82, 784-792.

11. Tahvanainen, H.; Pätynen, J.; Lokki, T. Studies on the perception of bass in four concert halls. Psychomusicology Music. Mind Brain 2015, 25, 294-305. [CrossRef]

12. Davies, W.J.; Cox, T.J. Response to "Comment on 'Reducing seat dip attenuation". J. Acoust. Soc. Am. 2001, 110, 1261-1262. [CrossRef]

13. LoVetri, J.; Mardare, D.; Soulodre, G. Modeling of the Seat-dip Effect Using the Finite-difference Time-domain Method. J. Acoust. Soc. Am. 1996, 100, 2204-2212. [CrossRef]

14. Sakurai, Y.; Morimoto, H.; Ishida, K. The reflection of sound transmission at grazing angles by auditorium seats. Appl. Acoust. 1993, 39, 209-227. [CrossRef]

15. Pätynen, J.; Tervo, S.; Lokki, T. Analysis of concert hall acoustics via visualisations of time-frequency and spatiotemporal responses. J. Acoust. Soc. Am. 2013, 133, 842-857. [CrossRef]

16. Tahvanainen, H.; Haapaniemi, A.; Lokki, T. Perceptual significance of seat-dip effect related direct sound coloration in concert halls. J. Acoust. Soc. Am. 2017, 141, 1560-1570. [CrossRef] [PubMed]

17. Ishida, K. The Measurement and Prediction of Sound Transmission over Auditorium Seats. Ph.D. Thesis, University of Cambridge, Cambridge, UK, 1993.

18. Tahvanainen, H.; Lokki, T.; Jang, H.-S.; Jeon, J.-Y. Investigating the influence of seating area design and enclosure on the seat-dip effect using scale model measurements. Acta Acust. 2020, 4, 15. [CrossRef]

19. COMSOL. Available online: cn.comsol.com (accessed on 3 May 2021).

20. Min, H.; Guo, W. Sound absorbers with a micro-perforated panel backed by an array of parallel-arranged sub-cavities at different depths. Appl. Acoust. 2019, 149, 123-128. [CrossRef]

21. Liu, C.R.; Wu, J.H.; Ma, F.; Chen, X.; Yang, Z. A thin multi-order Helmholtz metamaterial with perfect broadband acoustic absorption. Appl. Phys. Express 2019, 12, 084002. [CrossRef] 